\title{
MILLIMETER-WAVE OBSERVATIONS OF DIFFUSE CLOUDS
}

\author{
R. LUCAS \\ IRAM, Domaine Universitaire de Grenoble \\ 38406 St Martin D'Hérès, France \\ AND \\ H. S. LISZT \\ NRAO, Charlottesville, VA 22903, USA
}

\begin{abstract}
Using the IRAM instruments (interferometer on Plateau de Bure and 30-m telescope on Pico Veleta) we have made numerous observations of molecular absorption lines in front of extragalactic millimeter wavelength radio sources. Observations of $\mathrm{HCO}^{+}, \mathrm{CO}$ and $\mathrm{OH}$ show that the lines of sight studied in this way sample the outer edges of molecular clouds or the diffuse clouds with highest column densities. Collisional excitation of the rotational levels is not significant in this density range for most molecular species, and accurate column densities may be derived by assuming radiative equilibrium with the cosmic microwave background. Using this technique we have measured column densities of $\mathrm{CO}, \mathrm{HCO}^{+}$, $\mathrm{H}_{2} \mathrm{CO}, \mathrm{CN}, \mathrm{HCN}, \mathrm{HNC}, \mathrm{CS}, \mathrm{SO}, \mathrm{H}_{2} \mathrm{~S}, \mathrm{C}_{2} \mathrm{H}$, and $\mathrm{C}_{3} \mathrm{H}_{2}$ in several lines of sight, intersecting about 20 individual clouds with $\mathrm{CO}$ column densities in the range $210^{14}$ to $\sim 10^{16} \mathrm{~cm}^{-2}$. These results confirm that complex molecules achieve dark-cloud abundances at low extinctions, either by formation in the gas phase or on grains.
\end{abstract}

\section{Introduction}

Interstellar molecules were first detected in diffuse clouds, by means of optical absorption lines: $\mathrm{CH}, \mathrm{CN}$ and $\mathrm{CH}^{+}$were found in the 1930s. Other abundant molecules such as $\mathrm{H}_{2}, \mathrm{HD}, \mathrm{CO}$ and $\mathrm{OH}$ were found in the UV in the 1970s. A few other molecules have been detected there by $\mathrm{cm}$-wave absorption: $\mathrm{OH}, \mathrm{CH}, \mathrm{H}_{2} \mathrm{CO}, \mathrm{C}_{3} \mathrm{H}_{2}, \mathrm{NH}_{3}$. Some may also be found by their $\mathrm{mm}$-wave emission, though the lines are very weak: $\mathrm{CO}, \mathrm{CS}, \mathrm{CN}$, and most 
recently, $\mathrm{HCO}^{+}$. Due to the low densities, collisional excitation of molecular levels above the cosmic microwave background temperature is indeed expected to be very low, and line emission is a poor tool to study the molecular composition of diffuse clouds. Absorption lines, on the other hand, provide a direct measurement of molecular column densities, unaffected by the bias of collisional excitation. The rotational level populations are in equilibrium with the cosmic background radiation, which makes accurate determination of column densities possible with the detection of even a single line.

The choice of background sources for mm-wave absorption is rather limited, since few strong continuum sources are available. Galactic sources such as Cas A , Sgr B2, W49, have been used (Encrenaz et al., 1980; Greaves et al., 1992; Tieftrunk et al., 1994), but they lead to some difficulties: there is in most cases some emission linked to the sources, since they are HII regions or supernova remnants, associated with massive star-forming regions and giant molecular clouds, and it is difficult to disentangle emission and absorption (Liszt \& Lucas, 1995). We felt safer relying on extragalactic objects. The background sources are then quasars or active galactic nuclei, with very small angular size (most are studied with VLBI techniques). Though these sources are weaker (a few Janskys), interpreting the results is much easier: there is no bias in favor of high excitation regions, since random clouds are observed, and the emission is weak or absent (except for the CO molecule).

If any emision is seen, as is the case for $\mathrm{CO}$, interferometer observations are preferred to resolve out the emission. Then, either single dish emission or other absorption lines must be observed to determine the excitation temperatures.

\section{2. $\mathrm{HCO}^{+}, \mathrm{CO}, \mathrm{H}_{2} \mathrm{CO}$, and $\mathrm{OH}$}

Lucas \& Liszt (1996) have searched for $3 \mathrm{~mm} \mathrm{HCO}^{+} J=1-0$ absorption along 30 lines of sight toward extragalactic sources, with the Plateau de Bure Interferometer. They found many lines: all lines of sight with galactic latitude below $b=15^{\circ}$ (but one) were detected, some with numerous velocity components. Such absorption lines occur approximately $30 \%$ as often as $21 \mathrm{~cm}$ absorption by $\mathrm{HI}$, and optical depth distributions for $\mathrm{HCO}^{+}$and $\mathrm{HI}$ have very similar slopes. However only rare and weak $\mathrm{HCO}^{+}$emission was detected, implying that the excitation temperatures $T_{\mathrm{EX}}$ are very close to $2.73 \mathrm{~K}$.

We have recently extended this data set by observing $\mathrm{CO}$ absorption with the Plateau de Bure in some of these directions, at both $115 \mathrm{GHz}$ and $230 \mathrm{GHz}$ when the $1 \mathrm{~mm}$ source flux was strong enough. Part of the data is shown in Fig. 1. CO emission profiles have been obtained for all these 
directions with the NRAO 12m Kitt Peak antenna.

$\mathrm{CO}$ excitation temperatures may be derived in two ways: (i) by comparing emission and absorption in the same direction, assuming that the average line parameters are the same in the $\left(\sim 1^{\prime}\right)$ single dish emission beam and in the small solid-angle defined by the background source; (ii) by comparing optical depths in the $J=1-0$ and $J=2-1$ absorption lines. The latter way is preferable since the same volume of space is sampled in the two absorption measurements, but is restricted to the strongest $1 \mathrm{~mm}$ continuum sources.

The resulting excitation temperatures are in the range 2.7 to $6.5 \mathrm{~K}$, implying pressures $n_{\mathrm{H}_{2}} T_{\mathrm{K}}$ of order 3000 to $15000 \mathrm{~cm}^{-3} \mathrm{~K}$ (assuming $T_{\mathrm{K}}$ larger than about $10 \mathrm{~K}$ ). Using these excitation temperatures one may derive $\mathrm{CO}$ column densities, which we have plotted versus $\mathrm{HCO}^{+}$column densities on Fig. 2. The CO column densities fall between $10^{14}$ and $10^{16} \mathrm{~cm}^{-2}$, which is the column density range where $\mathrm{CO}$ self-shielding against photodissociation becomes important and $\mathrm{CO}$ begins to take up a large fraction of the gasphase carbon (see e.g. van Dishoeck \& Black, 1986). This transition occurs for $\mathrm{H}_{2}$ column densities around $10^{21} \mathrm{~cm}^{-2}$; it may also be present in Fig. 2 as an abrupt increase in $N(\mathrm{CO}) / N\left(\mathrm{HCO}^{+}\right)$around $N_{\mathrm{HCO}^{+}}=10^{12} \mathrm{~cm}^{-2}$, similar to that which we showed earlier (Lucas \& Liszt, 1996) for the CO emission brightness. Formaldehyde, observed by Liszt \& Lucas (1994) is also only present for column densities larger than $N_{\mathrm{HCO}^{+}} \sim 10^{12} \mathrm{~cm}^{-2}$; above this value $\mathrm{H}_{2} \mathrm{CO}$ is present with dark cloud-like abundances.

In front of a few sources ${ }^{13} \mathrm{CO}$ could be detected in absorption, in velocity components where the ${ }^{12} \mathrm{CO}$ optical depth is not so high as to prevent a determination of the ${ }^{12} \mathrm{CO}$ column density. We find (Fig. $2 \mathrm{~b}$ ) that this ratio varies between $\sim 15$ and $\sim 100$. Isotope-selective photodissociation tends to increase the ${ }^{12} \mathrm{CO}$ to ${ }^{13} \mathrm{CO}$ ratio, since self-shielding does not occur for the isotopic species, but in no case do we see ratios higher than the Solar value of 89. At higher column densities, the chemical fractionation reaction involving isotope exchange with $\mathrm{C}^{+}$(Watson, Anicich \& Huntress, 1976) seems to have displaced the isotopic abundance ratio to rather small values, an indication that fairly low $(<50 \mathrm{~K})$ kinetic temperatures occur even $N\left(\mathrm{C}^{+}\right) / N(\mathrm{CO})$ is still large.

We have observed $18 \mathrm{~cm}$ wavelength $\mathrm{OH}$ absorption and emission in some of these directions using the VLA, the $43 \mathrm{~m}$ NRAO telescope, and the Nançay telescope. A remarkably uniform relationship is found: the ratio of $\mathrm{HCO}^{+}$to $\mathrm{OH}$ ranges between 0.03 and 0.05 for $\mathrm{OH}$ column densities in the range $10^{12}$ to $2 \times 10^{13} \mathrm{~cm}^{-2}$ (Lucas \& Liszt, 1996; Liszt \& Lucas, 1996). This ratio is similar to the one found in dark clouds such as TMC1. One expects these two species to be related by means of the chemical reaction $\mathrm{C}^{+}+\mathrm{OH} \rightarrow \mathrm{CO}^{+}+\mathrm{H}$, leading to $\mathrm{HCO}^{+}$by reaction of $\mathrm{CO}^{+}$with $\mathrm{H}_{2}$. 

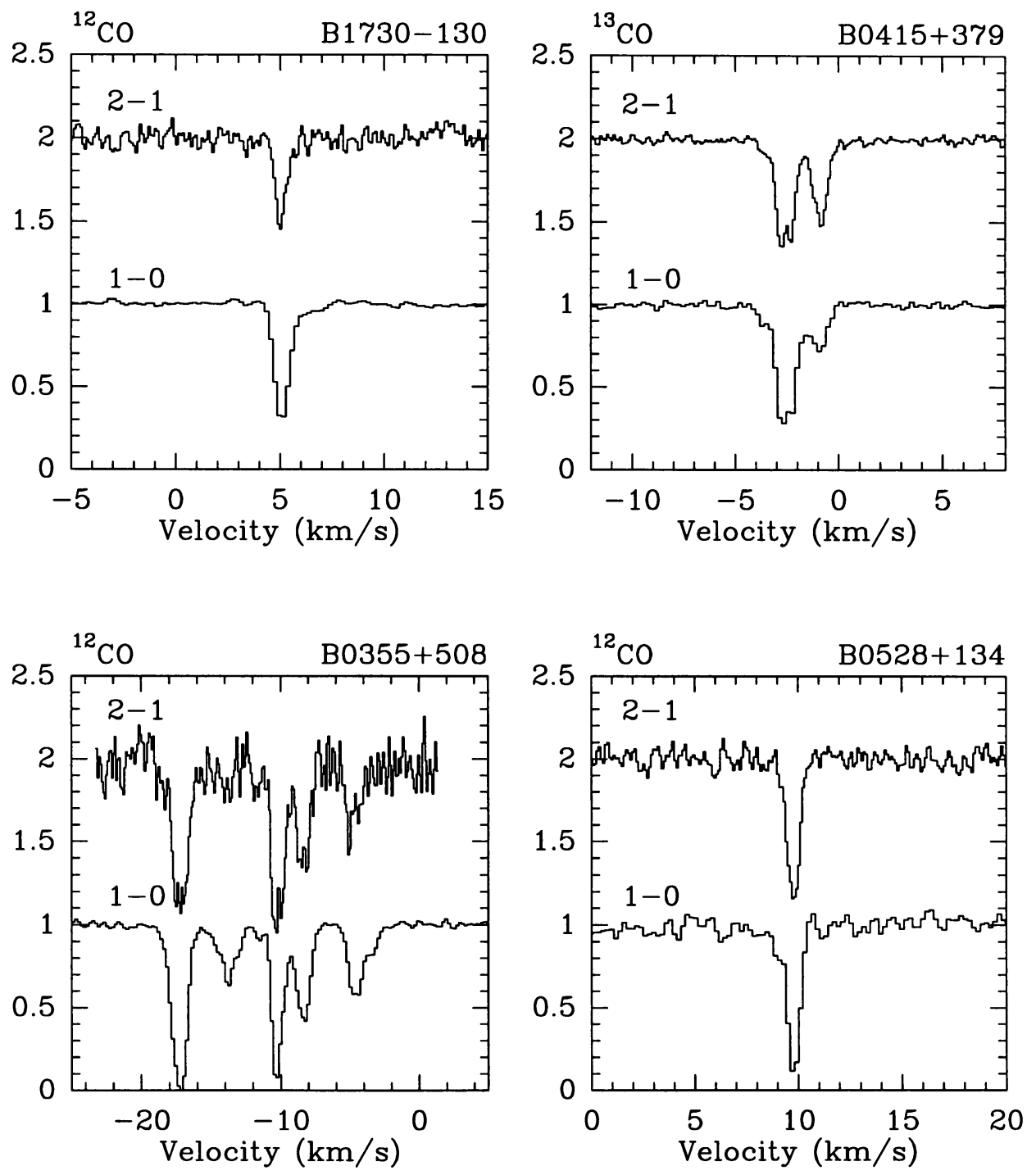

Figure 1. Comparison of CO 1-0 and 2-1 absorption profiles, observed with the Plateau de Bure interferometer. The $2-1$ spectra have been displaced vertically by +1 for clarity.

However this leads to $\mathrm{HCO}^{+}$to $\mathrm{OH}$ ratios which are too low by an order of magnitude.

Falgarone et al. (1995) have proposed a new chemical model of diffuse clouds involving the intermittent dissipation of supersonic turbulence, to produce $\mathrm{CH}^{+}$(and $\mathrm{HCO}^{+}$) by means of endothermic reactions (see also the models by Spaans, 1995); but these models produce also too much $\mathrm{OH}$, as compared to $\mathrm{HCO}^{+}$. Models involving MHD shocks (Pineau des 
(a)

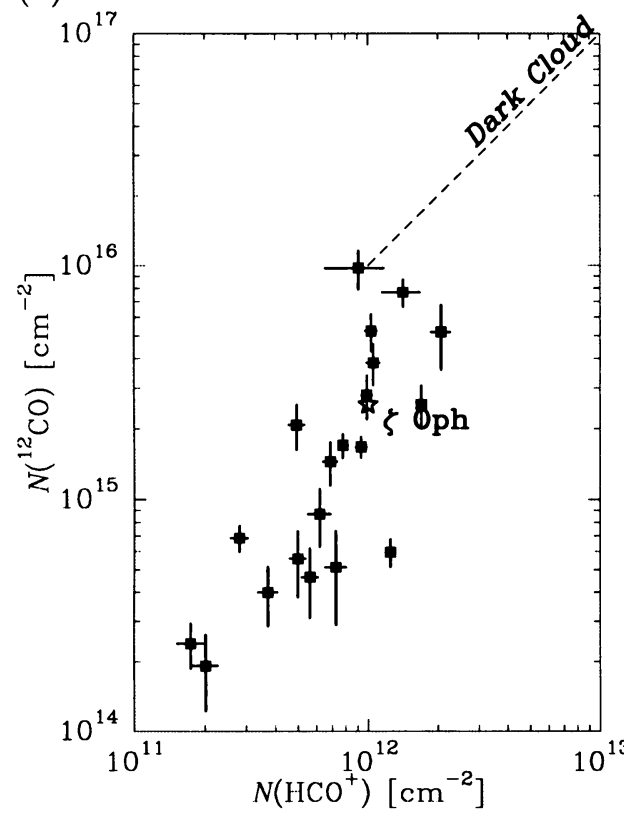

(b)

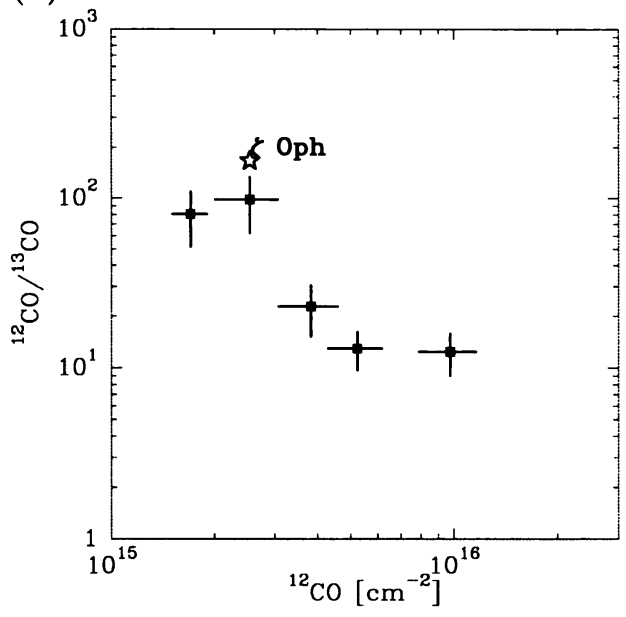

Figure 2. (a) $\mathrm{CO}$ versus $\mathrm{HCO}^{+}$column densities, from absorption-line measurements. The star indicates the values found in front of $\zeta$ Oph (Liszt and Lucas 1994); (b) The ${ }^{13} \mathrm{CO}$ to ${ }^{12} \mathrm{CO}$ isotopic ratio, as derived from absorption lines, as a function of ${ }^{12} \mathrm{CO}$ column density.

Forêts, private communication) can account for the observed ratio, but predict velocity differences of a few $\mathrm{km} / \mathrm{s}$ between $\mathrm{OH}$ and $\mathrm{HCO}^{+}$, which are not observed. Federman \& Williams (1996) claim that dissipation of MHD turbulence can produce large $\mathrm{HCO}^{+}$abundances without over-producing $\mathrm{OH}$.

\section{Other molecules}

\section{1. $\mathrm{HCN}, \mathrm{HNC}, \mathrm{AND} \mathrm{CN}$}

$\mathrm{HCN}, \mathrm{HNC}$ and $\mathrm{CN}$ have been easily observed in absorption in front of BL Lac (Lucas \& Liszt, 1993); HCN was also detected in front of B0212+735, B0355+508 (Lucas \& Liszt, 1994) and B0528+134 (Hogerheijde et al., 1995). We have undertaken an absorption line study of this chemical group in front of seven sources (B0212+737, B0355+508, B0415+379, B0528+134, B1730-130, B2200+420, B2251+158). We detected HCN in all seven directions, $\mathrm{CN}$ and $\mathrm{HNC}$ in most.

The column densities for each velocity component are all computed assuming that the excitation temperatures are equal to that of the cosmic 
a

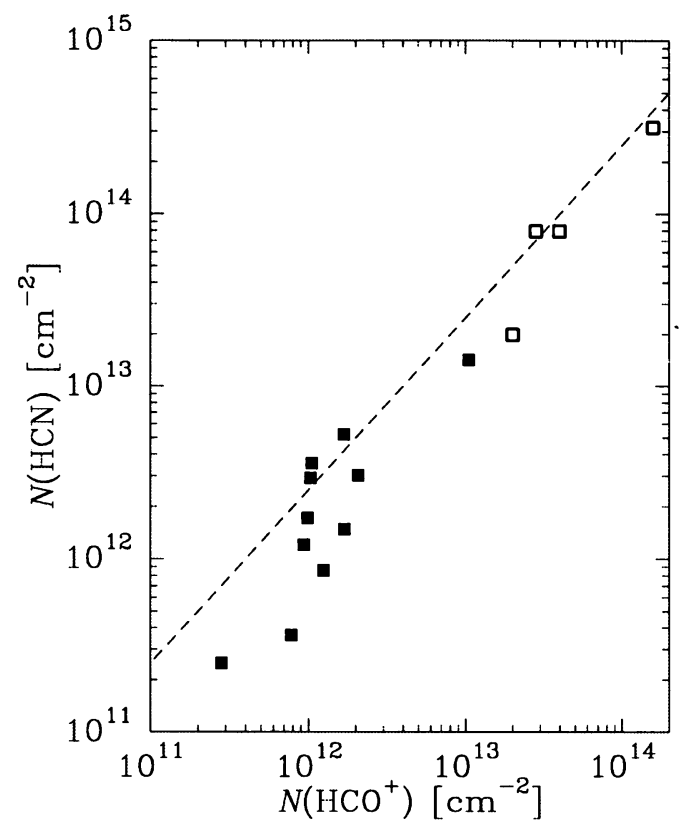

b

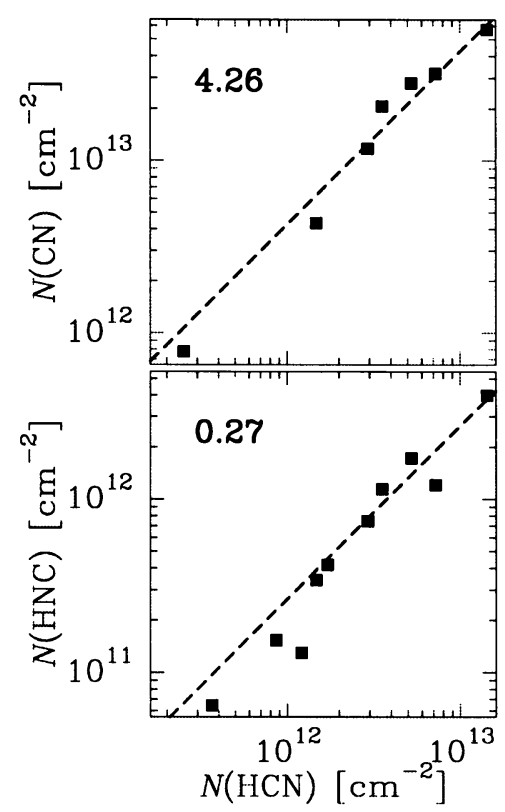

Figure 3. (a) $\mathrm{HCN}$ column density vs. $\mathrm{HCO}^{+}$column density. Filled squares: our data; open squares: data quoted by Tieftrunk et al. (1994). The dashed line indicates a typical dark cloud abundance ratio (TMC1). (b) Column densities of CN and HNC plotted vs. column density of HCN. The dashed lines indicate the average ratios.

background. The $\mathrm{HCN}$ to $\mathrm{HCO}^{+}$abundance ratio we find is about 2 , just as in dark clouds, but seems lower when $N\left(\mathrm{HCO}^{+}\right) \sim 10^{12} \mathrm{~cm}^{-2}$ (Fig. 3a). The $\mathrm{CN}$ and HNC column densities are compared to those of HCN in Fig. 3b. It is apparent that the ratios of $\mathrm{CN}$ and $\mathrm{HNC}$ relative to $\mathrm{HCN}$ have well defined values of about 4 and 0.3 . There is clearly more CN and less HNC than in dark clouds. Low HNC/HCN abundance ratios in molecular clouds seem to characterize warm temperature regions, as opposed to cold dark clouds, where this ratio is close to 1 . Our results on $\mathrm{CN}$ are compatible with the results of Federman et al. (1984) who found a strong variation of CN abundance with $\mathrm{H}_{2}$ column density, with a minimum $\mathrm{CN}$ column density of about $10^{12} \mathrm{~cm}^{-2}$.

\section{2. $\mathrm{C}_{2} \mathrm{H}$ AND $\mathrm{C}_{3} \mathrm{H}_{2}$}

We have also observed two hydrocarbons, $\mathrm{C}_{2} \mathrm{H}$ and $\mathrm{C}_{3} \mathrm{H}_{2}$ (Fig. 4 ). $\mathrm{C}_{2} \mathrm{H}$ is found to be much more abundant (relative to both $\mathrm{CO}$ and $\mathrm{HCO}^{+}$) than in dark clouds. In fact $\mathrm{C}_{2} \mathrm{H}$ seems even more widely distributed than $\mathrm{HCO}^{+}$. However, the abundance ratio of $\mathrm{C}_{2} \mathrm{H}$ to $\mathrm{CO}$ varies a lot from cloud to cloud, 


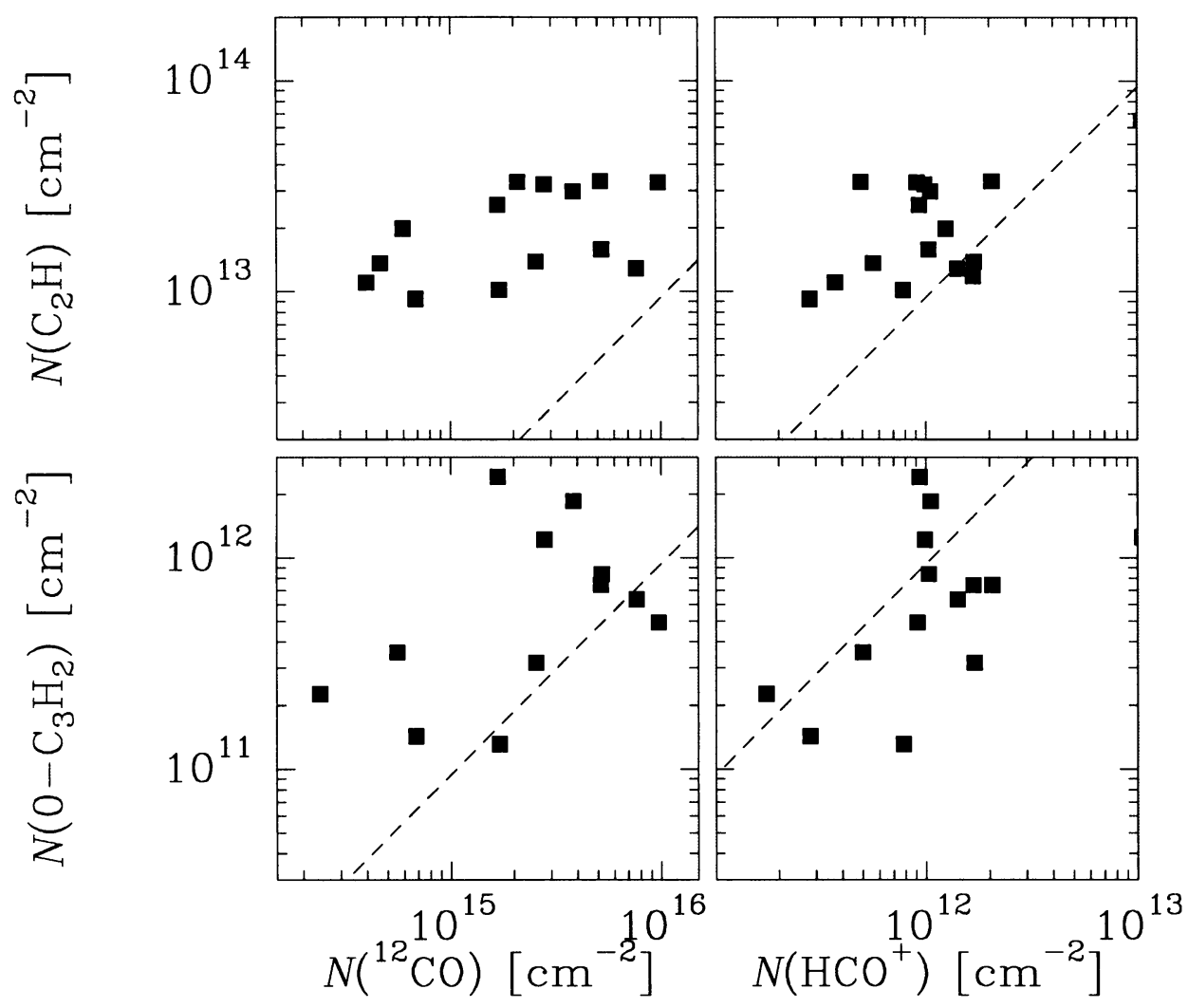

Figure 4. Column densities of $\mathrm{C}_{2} \mathrm{H}$ and $\mathrm{C}_{3} \mathrm{H}_{2}$ vs. column densities of ${ }^{12} \mathrm{CO}$ and $\mathrm{HCO}^{+}$. The dashed lines indicate typical dark cloud abundance ratios (TMC1).

ranging between 0.001 and 0.045 . Comparison with the optical results of Federman et al. (1984) for $\mathrm{CN}$ shows that our $\mathrm{C}_{2} \mathrm{H}$ column densities are typically 2-3 times lower than the $\mathrm{C}_{2}$ and $\mathrm{CH}$ column densities in front of stars with similar $\mathrm{CO}$ and $\mathrm{CN}$ column densities. The abundance of $\mathrm{C}_{3} \mathrm{H}_{2}$ (about $0.03 \%$ of that of $\mathrm{C}_{2} \mathrm{H}$ ), also shows much variation. This molecule had previously been observed in diffuse clouds by its centimeter-wave transitions (see e.g. Cox et al., 1988).

Are these molecules coming from grains or PAH's ? Pat Thaddeus suggested to us in 1995 May that we might be seeing different chemistries; one, exemplified by $\mathrm{OH}, \mathrm{HCO}^{+}$, etc. built up in the traditional way through gasphase ion-molecule reactions, and another, seen in the hydrocarbons $\mathrm{C}_{2} \mathrm{H}$ and $\mathrm{C}_{3} \mathrm{H}_{2}$, which arise from the fragmentation of large molecules by interstellar UV radiation. 


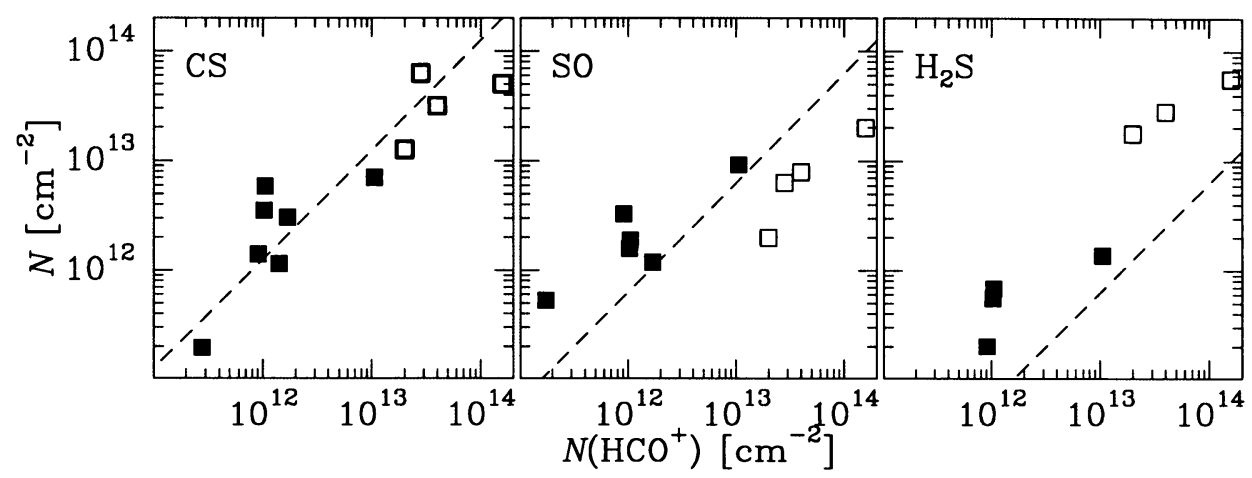

Figure 5. Column densities of sulphur compounds: CS, SO and $\mathrm{H}_{2} \mathrm{~S}$, plotted versus $\mathrm{HCO}^{+}$column density. Filled squares: our data; open squares: data quoted by Tieftrunk et al. (1994). The dashed lines indicate typical dark cloud abundance ratios (TMC1).

\subsection{CS, $\mathrm{SO}, \mathrm{AND} \mathrm{H}_{2} \mathrm{~S}$}

We have also detected $\mathrm{CS}$, SO, and $\mathrm{H}_{2} \mathrm{~S}$ (CS and SO with the Plateau de Bure, $\mathrm{H}_{2} \mathrm{~S}$ with the $30-\mathrm{m}$ telescope) in absorption. We find that CS has about the same abundance as in the 'spiral arm clouds' (Tieftrunk et al., 1994), while SO is more abundant by up to a factor of 10 (Fig. 5). In particular, SO tends to be as abundant as CS, or even more abundant, at the lowest column densities. $\mathrm{H}_{2} \mathrm{~S}$ is only slightly less abundant, on the average, than in the 'spiral arm clouds'.

The two most abundant sulphur-bearing molecules, CS and SO, are known to have highly variable relative abundances in molecular clouds. Le Bourlot et al. (1995), in particular, have shown that bistability in the chemical reactions may result in two different phases. The low ionization phase (LIP) should be rich in SO, while the high ionization phase (HIP) should have high CS and $\mathrm{HCS}^{+}$abundances, with vanishingly small $\mathrm{H}_{2} \mathrm{~S}$ and SO. Gérin et al. (1996) compared the abundances of CS, SO, $\mathrm{HCS}^{+}$ (and many other molecules) in regions which would be expected to have widely-differing cosmic ray ionization rates; the HIP and LIP could then occur in different proportions. Some aspects of the chemistry do indeed appear to reflect this segregation.

Tieftrunk et al. (1994) were not able to explain the large abundance of $\mathrm{H}_{2} \mathrm{~S}$ they observed in spiral arm clouds; Turner (1996) finds the same problem by measuring $\mathrm{H}_{2} \mathrm{~S}$ emission in translucent clouds. We find that this high abundance of $\mathrm{H}_{2} \mathrm{~S}$ persists to still lower column densities.

Spaans (1995) has performed model calculations of the lines of sights towards $\zeta$ Oph and o Per, including the effects of turbulence. He finds that indeed CS and SO have large and similar column densities (a few $10^{12}$ $\mathrm{cm}^{-2}$ ), but the predicted $\mathrm{H}_{2} \mathrm{~S}$ column densities are much lower than we 
have observed. $\mathrm{H}_{2} \mathrm{~S}$ could be enhanced by shocks (Pineau des Forêts et al., 1993) at high densities, or photodesorbed off grains in diffuse regions.

\section{Conclusions}

Millimeter wave absorption provides a way to measure accurately the molecular abundances in a selection of diffuse clouds. The results reveal that diffuse clouds have indeed a rich chemistry, although the UV field is strong. On the average abundance ratios are not so different from those in dark clouds. The photodissociation transition undertaken by $\mathrm{CO}$ is the most significant feature of these regions; some molecules such as $\mathrm{H}_{2} \mathrm{CO}$ or $\mathrm{HCN}$ seem to share this transition. However, species like $\mathrm{HCO}^{+}$and $\mathrm{OH}$ are still abundant in the $\mathrm{CO}$ poor regions and some molecules, such as hydrocarbons, are relatively more abundant. Furthermore, some order of magnitude variations in molecular abundances, expected when going from dark cloud chemistry to diffuse cloud chemistry, do not seem to occur.

\section{References}

Cox, P., Güsten, R., Henkel, C. 1988, A\&A 206, 108

Encrenaz, P.J., Combes, F., Lucas, R., Stark, A.A., Linke, R.A., Wilson, R.W., 1980, A\&A 88, L1

Falgarone, E., Pineau des Forêts, G., Roueff, E. 1995, A\&A 300, 870

Federman, S.R., Danks, A.C., Lambert, D.L. 1984, ApJ 287, 219

Federman, S.R., Williams, D. A. 1996, MNRAS, 279, L41

Gérin, M., Falgarone, E., Joulain, K., Kopp. M., Le Bourlot, J., Pineau des Forêts, G., Roueff, E., Schilke, P. 1996, A\&A, in press

Greaves J.S., Glenn, J.H., Ohishi, M., Hasegawa, T., Sunada, K. 1992, A\&A 260, 381

Hogerheijde, M.R., de Geus, E.J., Spaans, M., van Langevelde, H.J., van Dishoeck, E.F. 1995, ApJ 441, L93

Le Bourlot, J., Pineau des Forêts, G., Roueff, E. 1995, A\&A 297, 251

Liszt, H.S., Lucas, R. 1994, ApJ 431, L131

Liszt, H.S., Lucas, R. 1994, A\&A 299, 847

Liszt, H.S., Lucas, R. 1995, A\&A 294, 811

Liszt, H.S., Lucas, R. 1996, A\&A 314, 917

Lucas, R., Liszt, H.S. 1993, A\&A 276, L33

Lucas, R., Liszt, H.S. 1994, A\&A 282, L5

Lucas, R., Liszt, H.S. 1996, A\&A 307, 237

Pineau des Forêts, G., Roueff, E., Schilke, P., Flower, D.R. 1993, MNRAS 262, 915

Spaans, M., 1995, PhD Thesis, Rijkuniversiteit te Leiden, The Netherlands

Tieftrunk, A., Pineau des Forêts, G., Schilke, P., Walmsley, C.M. 1994, A\&A 289, 579

Turner, B. E. 1996, ApJ 468, 694

van Dishoeck, E.F., Black, J.H. 1986, ApJS 62, 109

Watson, W. D., Anicich, V., Huntress, W. T. 1976, ApJ 205, L165 


\section{Discussion}

Mauersberger: Toward one of the quasar absorption systems you report $\tau\left(\mathrm{C}^{18} \mathrm{O}\right)$ of 3 . How can one determine the column densities of CO?

Lucas: The $\mathrm{C}^{18} \mathrm{O}$ line is clearly saturated, and an upper limit to the optical depth is set by the negative result on the $\mathrm{C}^{17} \mathrm{O}$ line.

Roueff: You compare millimeter and centimeter observations. Are you sure that you sample the same area?

Lucas: The source sizes are very small at centimeter and millimeter wavelengths, although they tend to be larger at low frequencies. Of course the beams in emission are much larger than in absorption measurements.

Walmsley: Do you see any evidence in your data for high density clumps $\left(n_{H}>>\right.$ $1000 \mathrm{~cm}^{-3}$ ) such as the 1-100 AU type structures which seem to be required to account for time variations in absorption profiles or differences between profiles of components of double star systems?

Lucas: The constraint on density from the $\mathrm{CO}$ excitation that we deduce is $n_{\mathrm{H}_{2}} T_{k} \sim 10^{4} \mathrm{~cm}^{-3} \mathrm{~K}$. This calls for low densities on average (a few hundreds $\mathrm{cm}^{-3}$ at most). On the other hand we have a program to search for time variations that would be due to high density clumps drifting through the lines of sight, as seen by Marsher et al. by means of $\mathrm{H}_{2} \mathrm{CO}$ absorption. 